\title{
Controlling the synthesis of metal-organic framework UiO-67 by tuning its kinetic driving force
}

\author{
Gurpreet Kaur, ${ }^{\mathrm{a}}$ Sigurd Øien-Ødegaard, ${ }^{\mathrm{a}}$ Andrea Lazzarini, ${ }^{\mathrm{a}}$ Sachin Maruti Chavan, ${ }^{\mathrm{c}}$ Silvia Bordiga,,${ }^{\mathrm{a}} \mathrm{b}$ \\ Karl Petter Lillerud ${ }^{a}$ and Unni Olsbye ${ }^{a *}$
}

${ }^{a}$ Department of Chemistry, Center for Materials Science and Nanotechnology, University of Oslo, P.O. Box 1033, N-0315

Oslo, Norway

bepartment of Chemistry, NIS and INSTM Reference Centre, University of Torino, Via G. Quarello 15, 10135 Torino, Italy

'ProfMOF A/S, Kirkegårdsveien 45, 3616 Kongsberg, Norway

\begin{abstract}
The successful synthesis of metal-organic framework (MOF) compounds relies on an intricate interplay between the components of the synthesis liquor at the given synthesis conditions. Herein, we explore the interdependence of modulator, linker and solvent amounts in the synthesis of the Zr-based MOF, UiO-67. Our study suggests that control of linker vacancy defects in UiO67 is feasible by tuning the ratios of these components, and that such control derives from recognizing the kinetic driving forces during MOF crystal growth. Importantly, we show that linker vacancy defects (and modulator molecules occupying linker sites) can be reduced by limiting the solvent amount to maintain a saturated concentration of linker throughout the synthesis. The method enables formation of UiO-67 with an ideal 1:1 ratio between $\mathrm{Zr}$ and the 4,4'-biphenyldicarboxylic acid linker, without surplus linker in the mother liquor nor additional post-synthetic steps, and reduces the amount of dimethyl formamide (DMF) solvent to less than $20 \%$ of previously reported procedures.
\end{abstract}

Metal-organic frameworks (MOFs) are emerging as a potent class of materials as heterogeneous catalysts, adsorbents and sensors due to their structural diversity, tunable porosity and potential to include a wide range of active sites..$^{1}$ Importantly, such active sites can be incorporated into the structures of MOFs in well-controlled and verifiable ways.

The UiO family of $\mathrm{Zr}-\mathrm{MOF}$, where $\mathrm{Zr}_{6}(\mathrm{OH})_{4} \mathrm{O}_{4}{ }^{12+}$ clusters are 12-connected by organic dicarboxylate linkers to form a MOF framework with fcc structure, are known for their exceptional stability and high tolerance for structural defects. ${ }^{2}$ This topic has been investigated extensively for the parent UiO-66 material, with terephthalate linkers. ${ }^{3-5}$ The types and concentration of defects available in UiO-66 are well understood, and methods of synthesis and post-synthetic modifications exist to obtain UiO-66 with desired material properties (e.g. lewis acidity, stability, porosity). ${ }^{6-9}$

Among the prime MOF candidates as heterogeneous catalysts is the isoreticular UiO-67, with 4,4'-biphenyl dicarboxylate (bpdc) linkers. ${ }^{10} \mathrm{UiO}-67$ is highly thermally and chemically stable, and may incorporate a wide range of catalytically active ligands as part of its structure. ${ }^{11-13}$ The relationship between synthesis parameters and MOF properties has not been investigated to the same degree for UiO-67 as for UiO-66, and reported methods show poor reproducibility in terms of the porosity and stability of the product MOF. ${ }^{14-16}$ The stoichiometry of the non-defective (ideal) UiO-67 MOF dictates a 1:1 molar ratio between $\mathrm{Zr}$ and the linker. However, the typical linker occupancy coefficient is observed to be less than 1 (determined by thermogravimetry) in our previously reported works. ${ }^{12,17}$ The predominant type of UiO-67 defects that is reported in literature is linker vacancies (often referred to as "missing linker" defects). ${ }^{18}$ Vacant sites may be occupied by monocarboxylates (introduced as additives or by solvent hydrolysis, see below), inorganic anions (from the $\mathrm{Zr}$ precursor) or water/hydroxide pairs (from water). ${ }^{5,19}$

In the synthesis of UiO-67 (and other Zr-based MOFs), monocarboxylic acids are commonly added as structure directing "modulators". ${ }^{20-22}$ Since they occupy the same sites as the linkers, it is natural to assume that an increase in modulator concentration will correlate with its incorporation in the MOF, hereby reducing linker incorporation. A common strategy to overcome linker vacancy defects is to add a stoichiometric excess of linker in the synthesis, shifting the driving force of the reaction towards a product with higher linker content. ${ }^{4}$ Although this strategy is effective, it automatically admits a loss of the most expensive reagent. Another effective strategy is to perform postsynthetic linker installation in the defect MOF, ${ }^{18}$ but it adds labor and waste.

The aim of the current contribution is to understand and control synthesis-property correlations of UiO-67 to the same level of detail as of UiO-66, and to enable more sustainable, reproducible synthesis of UiO-67 MOFs with targeted $\mathrm{Zr}$ :linker ratios - a prerequisite for their subsequent industrial use.

Conventional UiO-67 synthesis is carried out by solvothermal reaction of $\mathrm{ZrCl}_{4}: \mathrm{H}_{2}$ bpdc:DMF in a molar ratio of 1:1:300500 and crystallizing at $120{ }^{\circ} \mathrm{C}$. Under these conditions, the reagents dissolve completely, and upon MOF formation, the concentration of reagents in solution, drops. The linker can exist in its solid form, dissolved in the mother liquor or as part of the MOF structure (Eq. 1):

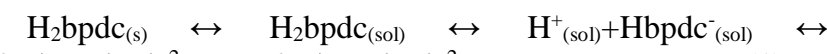
$2 \mathrm{H}^{+}{ }_{(\mathrm{sol})}+\mathrm{bpdc}^{2-}{ }_{(\mathrm{sol})} \leftrightarrow 2 \mathrm{H}^{+}{ }_{(\mathrm{sol})}+\mathrm{bpdc}^{2-}{ }_{(\mathrm{s}, \mathrm{MOF})}$

Addition of a modulator is required to form well-defined crystals of UiO-67. Behrens and co-workers reported poor UiO67 crystallinity below 5 eq of benzoic acid with respect to $\mathrm{Zr}$ 
content in DMF. ${ }^{22}$ Due to the common ion effect (in this case $\mathrm{H}^{+}$), modulator addition further decreases the already limited solubility of the $\mathrm{H}_{2}$ bpdc linker (Figure 1). Solvent hydrolysis represents a further complication; like the modulator, it adds to the $\mathrm{H}^{+}$content of solution, and the deprotonated formic acid competes with the linker for interaction with $\mathrm{Zr}^{4+}$ sites (Figure $1)$.

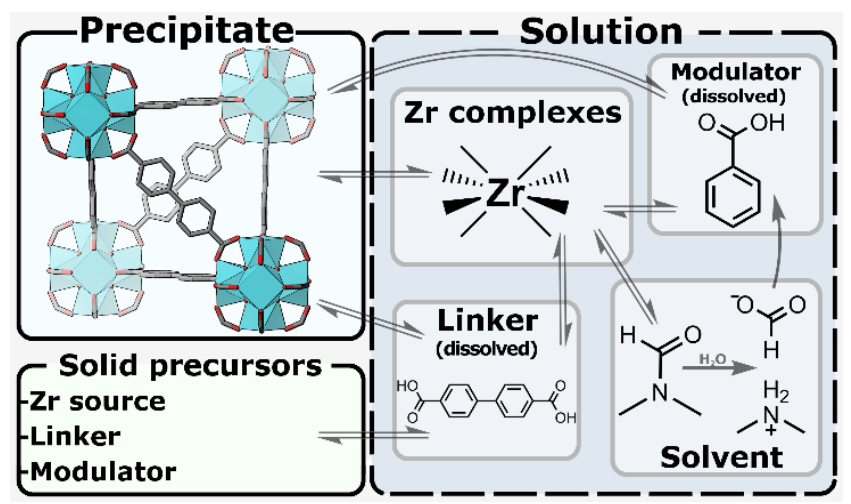

Figure 1. Reversible interactions (simplified) occurring during the formation of UiO-67.

Throughout MOF synthesis, the deprotonated analogues of linker, modulator and hydrolysed solvent participate in reversible interactions with the growing MOF crystallites, and therefore their concentration and affinity for the $\mathrm{Zr}_{\mathrm{x}}$ sites control the rate of nucleation, the rate of growth, and the fraction of defects in the final material (Figure 1). While modulator and solvent are present in excess, implying that their concentration changes little during MOF crystal growth, the linker is optimally present in stoichiometric ratio to $\mathrm{Zr}$. Hence, its concentration will decrease with MOF crystal growth, thereby increasing the probability of modulator- or decomposed linker-covered defect formation with time. Ultimately, the linker concentration becomes too low to compete with modulator and hydrolysed solvent for the $\mathrm{Zr}_{\mathrm{x}}$ sites, and crystal growth stops. This is addressed in timeresolved experiments performed by extracting the samples on intervals from the formation of UiO-67 and obtaining their composition with 1H-NMR (See section S17). A guiding hypothesis of this contribution is that maintenance of a stable linker concentration in the mother liquor until the $\mathrm{Zr}$ source is consumed is key to the formation of defect-free UiO-67, and may be achieved by tuning the initial Zr:linker:modulator:solvent ratios.

In the first part of this study, high-throughput screening synthesis was performed in order to define proper starting conditions for the subsequent, detailed investigations (Supporting Information, Sections S4). The screening studies led to the selection of $\mathrm{H}_{2} \mathrm{O}$ over aqueous $\mathrm{HCl}$, benzoic acid over acetic acid, a reaction temperature of $130{ }^{\circ} \mathrm{C}$, and $\mathrm{ZrCl}_{4}: \mathrm{H}_{2}$ bpdc: $\mathrm{H}_{2} \mathrm{O}: \mathrm{DMF}$ ratios of $1: 1: 3: 50$ (concentrated synthesis) or 1:1:3:300 (dilute synthesis) as starting conditions. As shown, concentrated synthesis enabled formation of defect-free $\mathrm{UiO}-67$, whereas $\mathrm{UiO}-$ 67 with linker vacancies was formed under dilute synthesis conditions.

It has recently been demonstrated that the amount of water also plays a major role in the synthesis of UiO-type MOFs. Behrens and coworkers observed that the rate of reaction is strongly correlated with increasing water content in the DMF-based modulated synthesis of UiO-66-fumarate ${ }^{23}$. Furthermore, Grey et al show that different phases can be obtained for the Hf analogue of UiO- 67 by changing the concentration of water. ${ }^{24}$ Even though we have kept the amount of water to be constant (3 eq) in all the experiments, it's worth mentioning that the amount of water can still vary in the reactions. DMF is a highly hygroscopic solvent, and thus the amount of water will vary slightly for dilute and concentrated syntheses. By Karl Fischer titration we have determined the average water content of the DMF used here is $0.06 \mathrm{wt} \%$, which translates to $0.7 \mathrm{eq}$ and 0.1 eq additional water in dilute synthesis and concentrated synthesis, respectively. As we did not observe different phases in the reported data, we consistently added 3 eq of water throughout the study, keeping in mind that the rate of the dilute reaction may be affected slightly more than the concentrated synthesis.

The core results of the study were obtained by two synthesis series in which the $\mathrm{Zr}$ :modulator ratio was systematically varied. These series of UiO-67 MOFs are labelled C-x $\mathbf{C}$ BA and D$\mathbf{X}_{\mathbf{B A}}$, signifying "concentrated" and "dilute", respectively in which $\mathrm{ZrCl}_{4}: \mathrm{H}_{2}$ bpdc: $\mathrm{H}_{2} \mathrm{O}: \mathrm{DMF}$ ratios of 1:1:3:50 and 1:1:3:300 were used. Under dilute synthesis conditions, a clear solution is obtained at the start of the synthesis. Under concentrated synthesis conditions, however, $\mathrm{ZrCl}_{4}$ and the modulator are fully soluble, while the linker is not. All reactions were stirred, as the concentrated synthesis protocol required it to dissolve linker as the reaction proceeded. In the respective concentrations, up to 18 molar equivalents of modulator (BA) with respect to reagents were used, and each material is labelled with this number. Thus, sample $\mathbf{C}-\mathbf{3}_{\mathbf{B A}}$ refers to $\mathrm{UiO}-67$ made in a concentrated synthesis with 3 molar equivalents of $\mathrm{BA}$, the ratios of $\mathrm{ZrCl}_{4}: \mathrm{H}_{2}$ bpdc:BA: $\mathrm{H}_{2} \mathrm{O}:$ DMF being 1:1:3:3:50 (see Table 1). Detailed synthesis conditions are described in SI, Section S5. Characterization data are shown in Figures 2-4, and in Figure S6.

Table 1. Summary of the main samples of this study, and their labels.

\begin{tabular}{|c|c|c|c|c|c|c|c|}
\hline \multirow{2}{*}{$\begin{array}{c}\text { Zr:DMF } \\
\text { ratio }\end{array}$} & \multicolumn{7}{|c|}{ Molar equivalents of BA in the synthesis } \\
\hline & 0 & 3 & 6 & 9 & 12 & 15 & 18 \\
\hline $1: 50$ & & $\mathrm{C}-3_{\mathrm{BA}}$ & C-6BA & $\mathrm{C}-9_{\mathrm{BA}}$ & $\mathrm{C}-12_{\mathrm{BA}}$ & C-15BA & C-18BA \\
\hline $1: 300$ & D-0BA & D-3BA & D-6BA & D-9 ${ }_{\mathrm{BA}}$ & D-12BA & D-15BA & D-18BA \\
\hline
\end{tabular}

Considering first the conventional, dilute synthesis (D-x $\mathbf{D A}$ series), Powder X-ray diffraction (PXRD) revealed that an increase in benzoic acid concentration ( $\mathrm{x}=0$ eq to $18 \mathrm{eq}$ ) leads to sharper and narrower diffraction peaks, indicating larger particle size and/or more ordered crystal structure (Figure 2a). This observation is in agreement with the findings of Behrens and coworkers. ${ }^{22}$ The average crystal size of the materials was estimated using peak fitting in TOPAS (Figure S6) ${ }^{25}$ and suggested increasing crystallite size for $\mathbf{D}-\mathbf{0}_{\mathbf{B A}}$ to $\mathbf{D}-\mathbf{9}_{\mathbf{B A}}$ and slightly decreasing size for higher BA contents. SEM micrographs confirmed the trend of increasing particle size with increasing amount of modulator (Figure 4). Furthermore, they revealed that samples D-0 $\mathbf{0}_{\mathbf{B A}}, \mathbf{D}-\mathbf{3}_{\mathbf{B A}}$ and $\mathbf{D}-\mathbf{6}_{\mathbf{B A}}$ also contains an unidentified phase of small particles (Figure 4).

Turning next to the concentrated synthesis series $\left(\mathbf{C}-\mathbf{x}_{\mathbf{B A}}\right)$, PXRD showed that pure UiO-67 is formed only in experiments C-3 $\mathbf{B A}_{\mathbf{B A}}, \mathbf{C - 6 \mathbf { B A }}$ and C-9 $\mathbf{B A}_{\mathbf{B A}}$ (Figure 2b). C-0 $\mathbf{B}_{\mathbf{B A}}$ contains UiO-67 as well as an unidentified crystalline phase indicated by the presence of extra peaks (for example at $2 \theta=12.7^{\circ}, 13.7^{\circ}$, and $14.3^{\circ}$ ). 
In C-12 BA $_{\text {and }} \mathbf{C - 1 5}$ BA, mainly linker with smaller amounts of UiO-67 is observed by XRD, presumably because the high concentration of benzoic acid lowers the solubility of $\mathrm{H}_{2}$ bpdc due to the common ion effect $\left(\mathrm{H}^{+}\right)$. Peak fitting in TOPAS indicated that the UiO-67 crystal size of the $\mathbf{C}$ - $\mathbf{x}_{\text {BA }}$ series increases slowly up to $\mathrm{x}=9$ and then rapidly decreases (Figure S6). SEM micrographs (Figure 4) depict well-defined homogeneous octahedral shaped crystals with particle size around $1.5 \mu \mathrm{m}$ for $\mathbf{C}-\mathbf{3}_{\mathbf{B A}}$. As the amount of benzoic acid increases to 6 eq and 9 eq in $\mathbf{C}-\mathbf{x}_{\mathbf{B A}}$, irregular particles with new facets (particularly [ $\left[\begin{array}{lll}1 & 0 & 0\end{array}\right]$ in addition to [ $\left.\left[\begin{array}{lll}1 & 1 & 1\end{array}\right]\right)$ are observed, as well as an increase in particle intergrowth and particle size dispersion (Figure 4 and Figure S13). Particle sizes for C-0BA, C-12 $\mathbf{B A}$ and $\mathbf{C - 1 5}$ Ba were not possible to determine. In spite of the well-crystalline UiO-67 phase indicated by XRD for C-0 $\mathbf{O}_{\mathbf{B A}}$, SEM did not reveal any particles with well-defined crystal morphology. C-12 ${ }_{\text {BA }}$ and C-15BA consist of irregular agglomerates without clear morphology, in line with XRD results.

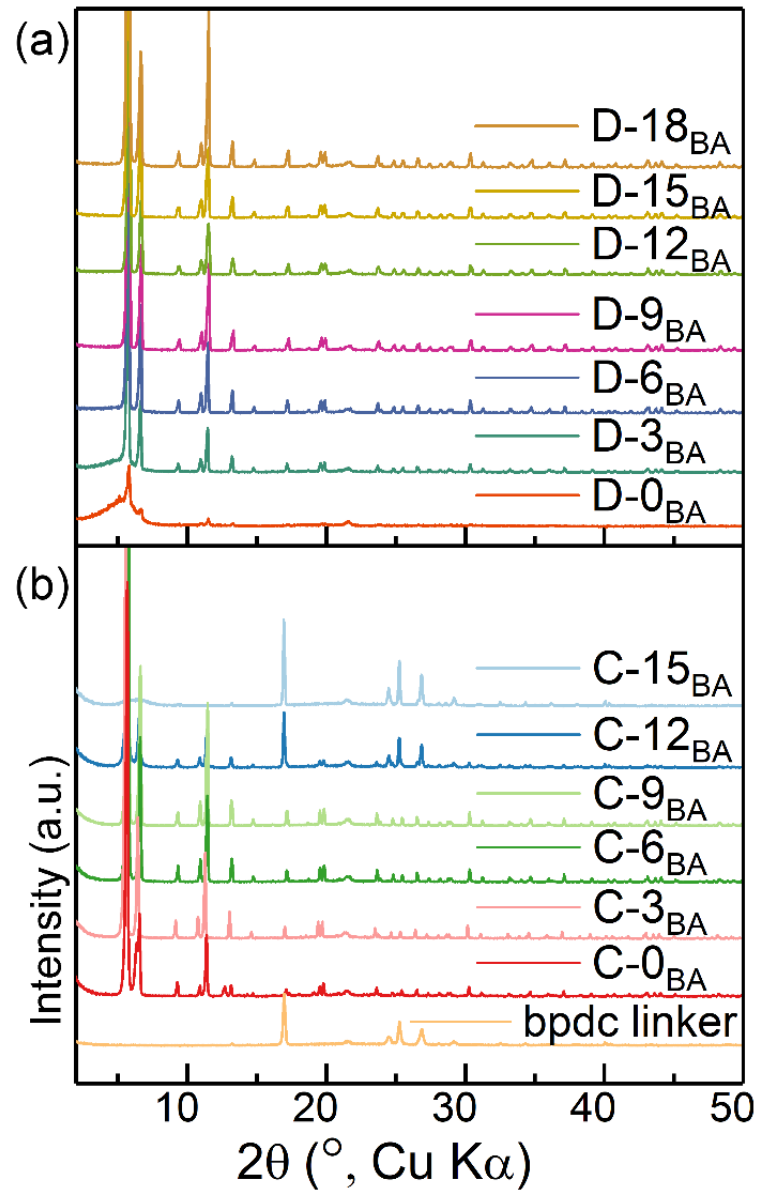

Figure 2. PXRD patterns of a) D-xBA (Zr:DMF ratio 1:300) and b) C-XBA (Zr:DMF ratio 1:50) series depicting the possibility to synthesize UiO-67 in the presence of various concentration of benzoic acid, $\mathrm{x}$ ( $\mathrm{x}=0$ to $18 \mathrm{eq}$ ).

The porosity of the $\mathbf{C}-\mathbf{x}_{\mathbf{B A}}$ and $\mathbf{D}-\mathbf{x}_{\mathbf{B A}}$ series was determined by nitrogen adsorption at $77 \mathrm{~K}$ (isotherms are shown in Figure $\mathrm{S} 12$ ). Figure $3 \mathrm{~b}$ ) shows the BET surface areas of the $\mathbf{C}$-хвA and D-xBa series, calculated from the nitrogen isotherms using established consistency criteria. ${ }^{26}$ The highest porosity is found for $\mathbf{C - 3} \mathbf{3}_{\mathbf{B A}}$, with a slight decrease as the amount of BA is increased. The BET surface area of $\mathbf{C - 3} \mathbf{B}_{\mathbf{B A}}\left(2734 \mathrm{~m}^{2} \mathrm{~g}^{-1}\right)$ is close to the computationally estimated UiO-67 surface area of 2700 $\mathrm{m}^{2} \mathrm{~g}^{-1}{ }^{4}$ In the case of the D-X $\mathbf{D A}$ series, the porosity increases abruptly with the amount of BA up till D-6BA, followed by a minor increase with further increase of BA.

In full agreement with the high specific surface areas, ATR analysis made on the pristine materials confirmed the presence of only a small amount of unreacted benzoic acid or dimethylformamide (broad, complex and very weak components around $1700-1730 \mathrm{~cm}^{-1}$, assigned to the peculiar $\mathrm{v}(\mathrm{C}=\mathrm{O})$ mode, shown in Figure S14). The same analyses suggest that the syntheses performed in absence of modulators $\left(\mathbf{C}-\mathbf{0}_{\mathbf{B A}}\right.$ and $\left.\mathbf{D - 0} \mathbf{0}_{\mathbf{B A}}\right)$ make materials with some impurities (please see the ATR spectra that are characterized by some extra peaks (Figure S14)).

DRIFT analysis was further performed after samples activation in helium at $300{ }^{\circ} \mathrm{C}$, followed by cooling to $70{ }^{\circ} \mathrm{C}$. The samples were monitored by DRIFT before and after $\mathrm{CD}_{3} \mathrm{CN}$ dosing. The results, illustrated in Figures S15 and S16 show that all the residual traces of unreacted modulator or DMF have been removed (absence of any band around $1700-1730 \mathrm{~cm}^{-1}$ ) and that all the samples are characterized by fully hydroxylated clusters (intense band at $3676 \mathrm{~cm}^{-1}$ ). Upon interaction with $\mathrm{CD}_{3} \mathrm{CN}$, the bands associated to the $\mathrm{OH}$ groups were eroded and shifted to lower frequencies. As counterpart, in the $v(\mathrm{CN})$ region (see the insets of Figures S15 and S16) new components around 2276 $\mathrm{cm}^{-1}$, are observed, while the absence of relevant bands around $2300 \mathrm{~cm}^{-1}$, exclude the abundance of accessible Lewis sites (e.g. uncoordinated $\mathrm{Zr}$ sites).

Thermogravimetric analysis (TGA, $30-800{ }^{\circ} \mathrm{C}$ ) was performed to determine the total organic content of the synthesized materials. At $800{ }^{\circ} \mathrm{C}$, in an oxidizing atmosphere we assume that only $\mathrm{ZrO}_{2}$ remains, and thus the TGA curves were normalized to this value (100\%). Two main weight losses are observed in the samples (Figure S7); near $200{ }^{\circ} \mathrm{C}$, corresponding to the removal of physisorbed solvent and modulator, and near $490^{\circ} \mathrm{C}$ with the combustion of the organic components of the material. The weight of the samples before dehydroxylation of the $\mathrm{Zr}_{6} \mathrm{O}_{4}(\mathrm{OH})_{4}{ }^{12+}$ cluster $\left(\right.$ at $200{ }^{\circ} \mathrm{C}$ ) and before linker combustion (at $400{ }^{\circ} \mathrm{C}$ ) were compared with the expected weight of the hydroxylated $(287 \%)$ and dehydroxylated $(282 \%)$ UiO-67, respectively, as calculated from the ideal structure (Section 10.1 in SI).

At $400{ }^{\circ} \mathrm{C}$, sample $\mathbf{C}-\mathbf{3}_{\mathbf{B A}}$ shows close to ideal relative mass of $282 \%$, whereas C-0ва, C-6Ba and C-9 $\%$ and $286 \%$, respectively (Figure S7a, S7b and S8). The corresponding figures of the $\mathbf{D}$-x $\mathbf{x A}$ samples were all in the range of $233-274 \%$, indicating a lower relative content of linker (Figure S7c and S9). The method relies on the assumption that the modulator content is negligible at $400{ }^{\circ} \mathrm{C}$. In case this assumption is incorrect (thus overestimating the linker content), the method was further developed to determine the identity of the organic components.

NMR spectroscopy of dissolved MOF samples was combined with the TGA-determined sample weights at $200{ }^{\circ} \mathrm{C}$ to estimate the total organic content in the samples. The main organic constituents in UiO-67 are bpdc ${ }^{2-}$, benzoate and formate (formic acid (FA); an in situ product of the hydrolysis of DMF) ${ }^{15}$ By treatment with NaOD (after heating the samples to $200{ }^{\circ} \mathrm{C}$ to remove the solvent), the carboxylates go into solution, and $\mathrm{Zr}$ precipitates as a mixed oxide/hydroxide. The composition of the liquid phase is then determined by ${ }^{1} \mathrm{H}-\mathrm{NMR}$ spectroscopy (see section 10.2 in SI for full details), and the molar ratios of the carboxylates are finally scaled to fit the relative 
mass of organics determined by TGA. The results from using both methods are shown in Figure $3 a$.

In the $\mathbf{C}$-Xвa series, a near ideal bpdc ${ }^{2-}$ to $\mathrm{Zr}$ ratio is observed in all samples $(x=0$ to 9$)$. A small increase of the benzoate content is observed when its concentration in the synthesis liquor is increased, yielding a total molar organic content that surpasses the $\mathrm{Zr}$ content. DRIFT studies suggest the absence of physisorbed modulator (see Figure S15-S16). Hence, the difference probably arises from cluster vacancy defects, causing a $\mathrm{Zr}$

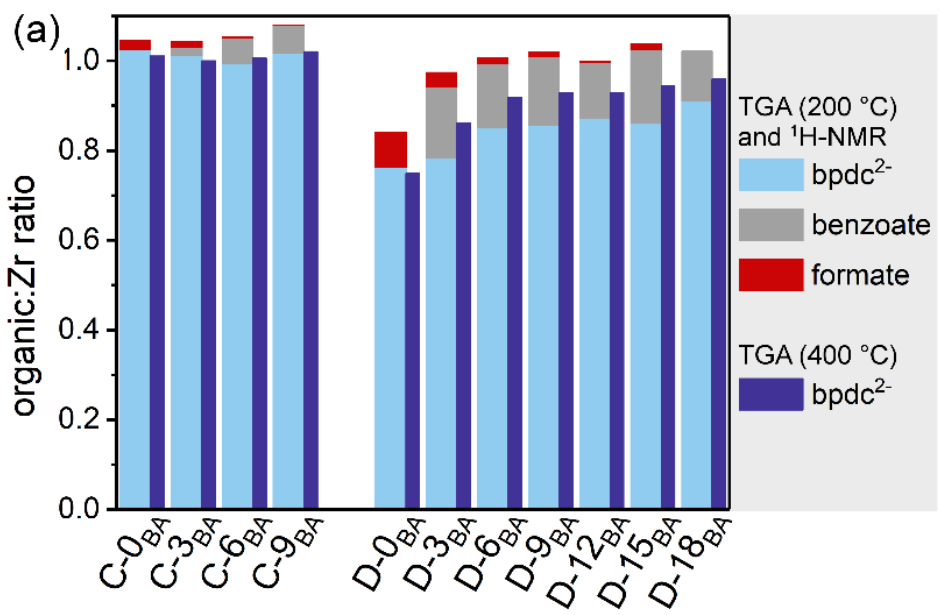

deficit, or termination of the outer surface of the MOF by organic carboxylate groups. The amount of benzoate present in C-9 ${ }_{\mathbf{B A}}$ is small; only $6 \%$ with respect to the amount of linker, compared to a 9:1 ratio between benzoate and linker in the synthesis liquor. Each linker vacancy defect can accommodate two monocarboxylates since each molecule occupies only one coor-

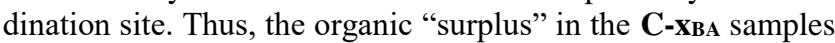
is 2-6\%. Importantly, the yield of concentrated synthesis, based on initial Zr content, was typically 86-92\% (Section S13).

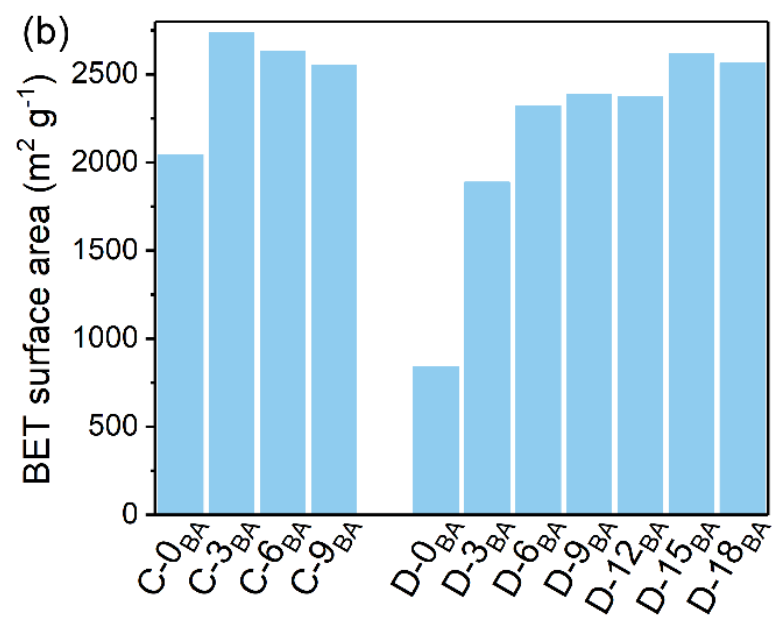

Figure 3. (a) Composition of the organic constituents in each sample (broad columns), determined by ${ }^{1} \mathrm{H}-\mathrm{NMR}$ and scaled to the organic: $\mathrm{Zr}$ ratio found by TGA (at $200{ }^{\circ} \mathrm{C}$ ), and the bpdc ${ }^{2-}: \mathrm{Zr}$ ratio found by TGA (at $400{ }^{\circ} \mathrm{C}$ ) only (thin columns) in the $\mathbf{C}$-xBA and $\mathbf{D}$-xBA series. The ratio in an ideal UiO-67 is 1. (b) BET Surface area of C- ХвA and D-хвA samples from $\mathrm{N}_{2}$ adsorption isotherms at $77 \mathrm{~K}$.

Strikingly, the D-x $\mathbf{x}_{\mathbf{B A}}$ series has a much lower bpdc ${ }^{2-}$ content, with high contents of benzoate $\left(15-20 \%\right.$ with respect to bpdc ${ }^{2-}$ ) and formate (as high as $10 \%$ with respect to bpdc ${ }^{2-}$ ) throughout the series. In the highly crystalline $\mathbf{D}-\mathbf{x}_{\mathbf{B A}}(\mathrm{x}=6$ to 15$)$ samples with high BET area the benzoate to linker ratio is rather high and constant, while for low modulator $\mathrm{x}=0$ and 3 , other constituents such as formic acid, $\mathrm{Cl}^{-}$and $\mathrm{OH}^{-}$seems to compete favorably with both benzoate and $\mathrm{bpdc}^{2-}$ for the $\mathrm{Zr}$ sites.

While there is good agreement between the two methods of determining the linker:Zr ratio in the $\mathbf{C}$-Xвa samples, there is a consistent disagreement concerning the D-x $\mathbf{x A}$ samples. These samples contain a lot of benzoate coordinated to $\mathrm{Zr}$ sites, and our results suggest that it cannot be readily assumed that all of this benzoate is removed once the sample is heated to $400{ }^{\circ} \mathrm{C}$. Overall, the amount of linker vacancy defects per $\mathrm{Zr}$ cluster is always $<1 / 12$ for the $\mathbf{C}$-XвA series while it varies from 2-5/12 for the D-x $\mathbf{D A}$ series (Figure 3a).

To assess the thermal stability of the optimal material, $\mathbf{C - 3}$-3A, in more detail, the sample was subject to static temperature treatment in air in the TGA instrument for $24 \mathrm{~h}$, followed by XRD analysis (Section S15). The XRD results are presented in Figure S17a, while TGA curves are presented in Figure S18. Together, the measurements reveal that the sample is stable at $350^{\circ} \mathrm{C}$, but slowly decomposes at temperatures above $350^{\circ} \mathrm{C}$.
In order to verify the hypothesis that linker concentration in the synthesis liquor is kept constant by the presence of its corresponding solid throughout MOF crystal formation, we performed a synthesis using the $\mathbf{C}-\mathbf{3}_{\mathbf{B} \text { A }}$ protocol, sampling the suspension 25 times during the first 12 hours of the synthesis (section S16). The solid fraction of each sample was isolated by filtration and PXRD patterns were acquired. We observed a gradual decrease in the diffraction pattern of $\mathrm{H}_{2} \mathrm{bpdc}_{(\mathrm{s})}$, until it disappeared after around 11 hours. It can be assumed that the solution is saturated by $\mathrm{H}_{2}$ bpdc in this period, due to the continuous agitation of the mixture. Simultaneously, the most intense diffraction peaks from UiO-67 (( $\left.\begin{array}{lll}1 & 1 & 1\end{array}\right)$ and $\left.\left(\begin{array}{lll}2 & 0 & 0\end{array}\right)\right)$ appeared as well-resolved peaks already after 45 minutes of reaction, and the reaction was mainly complete after $12 \mathrm{hrs}$. In a parallel experiment following the D-9 $\mathbf{B A}$ protocol, it was observed that benzoate concentration of the product increased along with the yield as the reaction progressed, indicating that linker incorporation in the MOF follows the waning concentration of linker in the mother liquor. Full details can be found in section 17 in SI.

The most commonly used variant of UiO-67 in heterogeneous catalysis is the one in which a fraction of the $\mathrm{H}_{2}$ bpdc linker is replaced by 2,2'-bipyridine-4,4'-dicarboxylic acid $\left(\mathrm{H}_{2}\right.$ bpydc). In a final synthesis effort, the concentrated synthesis protocol was applied to $\mathrm{UiO}-67$ with $10 \%$ bpydc. 


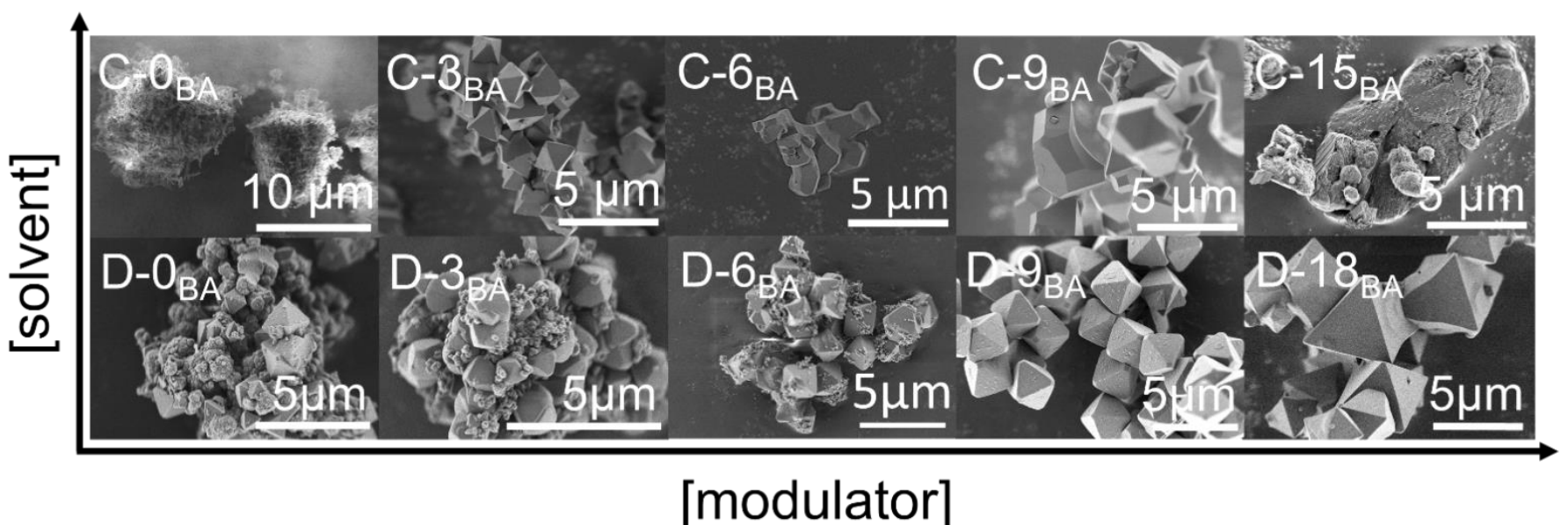

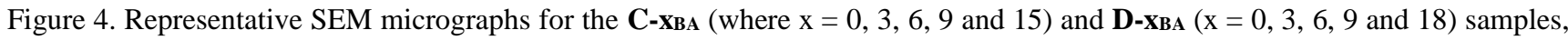
highlighting the effect of the concentrated and diluted synthesis procedure on the MOF particles.

The resulting material was characterized by XRD, TGA, NMR, BET and SEM, and revealed a highly crystalline, pure UiO-67-bpy 0.1 material with a $1: 1$ Zr:linker ratio and a bpdc:bpydc ratio of 0.9:0.1 (Section S18), thereby confirming the applicability of the concentrated synthesis protocol to functionalized UiO-67.

In conclusion, this study demonstrates that the amount of linker vacancy defects in UiO-67 can be controlled by tuning the ratios of reagents, modulator and solvent. Conventional approaches to UiO-67 synthesis yield materials with high concentration of linker vacancy defects, which may be desirable for catalytic purposes or as starting material for post-synthetic linker insertion. Near defect-free UiO-67 (i.e. negligible amount of missing linker defects, low amount of residual species, highly porous and well-defined particle morphology) can be produced by using a minimal amount of DMF, in combination with an optimal linker-to-modulator ratio, due to thorough balancing of the multiple reversible interactions taking place between the components of the synthesis liquor during UiO-67 formation. An added benefit of the concentrated solution method is the lowering of toxic waste to a fraction of previously reported methods.

\section{ASSOCIATED CONTENT}

Supporting Information. Preliminary screening of MOF synthesis conditions, detailed synthesis procedures, additional characterization data and detailed descriptions of chemicals, instrumentation and methods. This material is available free of charge via the Internet at http://pubs.acs.org.

\section{AUTHOR INFORMATION}

\section{Corresponding Author}

* Unni Olsbye. Email: unni.olsbye@kjemi.uio.no

\section{ACKNOWLEDGMENT}

The author thanks Erlend Aunan for calculating the water content in commercially obtained DMF using Karl Fischer titration. The Norwegian Research Council is acknowledged for financial support of this study through contract no. 250795 (CONFINE).

\section{References}

1. Furukawa, H.; Cordova, K. E.; O'Keeffe, M.; Yaghi, O. M., The Chemistry and Applications of Metal-Organic Frameworks. Science 2013, 341 (6149), 1230444.

2. Taddei, M., When defects turn into virtues: The curious case of zirconium-based metal-organic frameworks. Coordination Chemistry Reviews 2017, 343, 1-24.

3. Shearer, G. C.; Chavan, S.; Bordiga, S.; Svelle, S.; Olsbye, U.; Lillerud, K. P., Defect Engineering: Tuning the Porosity and Composition of the Metal-Organic Framework UiO-66 via Modulated Synthesis. Chemistry of Materials 2016, 28 (11), 3749-3761.

4. Shearer, G. C.; Chavan, S.; Ethiraj, J.; Vitillo, J. G.; Svelle, S.; Olsbye, U.; Lamberti, C.; Bordiga, S.; Lillerud, K. P., Tuned to Perfection: Ironing Out the Defects in Metal-Organic Framework UiO66. Chemistry of Materials 2014, 26 (14), 4068-4071.

5. Øien, S.; Wragg, D.; Reinsch, H.; Svelle, S.; Bordiga, S.; Lamberti, C.; Lillerud, K. P., Detailed Structure Analysis of Atomic Positions and Defects in Zirconium Metal-Organic Frameworks. Crystal Growth \& Design 2014, 14 (11), 5370-5372.

6. Shearer, G. C.; Vitillo, J. G.; Bordiga, S.; Svelle, S.; Olsbye, U.; Lillerud, K. P., Functionalizing the Defects: Postsynthetic Ligand Exchange in the Metal Organic Framework UiO-66. Chemistry of Materials 2016, 28 (20), 7190-7193.

7. Fei, H.; Pullen, S.; Wagner, A.; Ott, S.; Cohen, S. M., Functionalization of robust $\mathrm{Zr}(\mathrm{IV})$-based metal-organic framework films via a postsynthetic ligand exchange. Chem. Commun. 2014, Ahead of Print

8. Kim, M.; Cahill, J. F.; Su, Y.; Prather, K. A.; Cohen, S. M., Postsynthetic ligand exchange as a route to functionalization of 'inert' metal-organic frameworks. Chem. Sci. 2012, 3 (1), 126.

9. Garibay, S.; Cohen, S., Isoreticular synthesis and modification of frameworks with the UiO-66 topology. Chem. Commun. 2010, 46 (41), 7700-7702.

10. Cavka, J. H.; Jakobsen, S.; Olsbye, U.; Guillou, N.; Lamberti, C.; Bordiga, S.; Lillerud, K. P., A New Zirconium Inorganic Building Brick Forming Metal Organic Frameworks with Exceptional Stability. J. Am. Chem. Soc. 2008, 130, 13850-13851.

11. Wang, C.; Xie, Z.; deKrafft, K. E.; Lin, W., Doping MetalOrganic Frameworks for Water Oxidation, Carbon Dioxide Reduction, and Organic Photocatalysis. J. Am. Chem. Soc. 2011, 133 (34), 1344513454.

12. Øien-Ødegaard, S.; Bouchevreau, B.; Hylland, K.; Wu, L.; Blom, R.; Grande, C.; Olsbye, U.; Tilset, M.; Lillerud, K. P., UiO-67type Metal-Organic Frameworks with Enhanced Water Stability and Methane Adsorption Capacity. Inorganic Chemistry 2016, 55 (5), 1986-1991.

13. Øien, S.; Agostini, G.; Svelle, S.; Borfecchia, E.; Lomachenko, K. A.; Mino, L.; Gallo, E.; Bordiga, S.; Olsbye, U.; Lillerud, K. P.; Lamberti, C., Probing Reactive Platinum Sites in UiO- 
67 Zirconium Metal-Organic Frameworks. Chemistry of Materials 2015, 27 (3), 1042-1056.

14. Chavan, S.; Vitillo, J. G.; Gianolio, D.; Zavorotynska, O.; Civalleri, B.; Jakobsen, S.; Nilsen, M. H.; Valenzano, L.; Lamberti, C.; Lillerud, K. P.; Bordiga, S., H2storage in isostructural UiO-67 and UiO-66 MOFs. Physical Chemistry Chemical Physics 2012, 14 (5), $1614-1626$

15. Larabi, C.; Quadrelli, E. A., Titration of $\mathrm{Zr} 3(\mu-\mathrm{OH})$ Hydroxy Groups at the Cornerstones of Bulk MOF UiO-67, [Zr6O4(OH)4(biphenyldicarboxylate)6], and Their Reaction with [AuMe(PMe3)]. European Journal of Inorganic Chemistry 2012, 2012 (18), 3014-3022.

16. Schaate, A.; Roy, P.; Godt, A.; Lippke, J.; Waltz, F.; Wiebcke, M.; Behrens, P., Modulated Synthesis of Zr-Based MetalOrganic Frameworks: From Nano to Single Crystals. Chemistry - A European Journal 2011, 17 (24), 6643-6651.

17. Gutterød, E. S.; Øien-Ødegaard, S.; Bossers, K.; Nieuwelink, A.-E.; Manzoli, M.; Braglia, L.; Lazzarini, A.; Borfecchia, E.; Ahmadigoltapeh, S.; Bouchevreau, B.; Lønstad-Bleken, B. T.; Henry, R.; Lamberti, C.; Bordiga, S.; Weckhuysen, B. M.; Lillerud, K. P.; Olsbye, U., CO2 Hydrogenation over Pt-Containing UiO-67 ZrMOFs - The Base Case. Industrial \& Engineering Chemistry Research 2017, 56 (45), 13206-13218

18. Gutov, O. V.; Hevia, M. G.; Escudero-Adán, E. C.; Shafir, A., Metal-Organic Framework (MOF) Defects under Control: Insights into the Missing Linker Sites and Their Implication in the Reactivity of Zirconium-Based Frameworks. Inorganic Chemistry 2015, 54 (17), 8396-8400

19. Trickett, C. A.; Gagnon, K. J.; Lee, S.; Gándara, F.; Bürgi, H.-B.; Yaghi, O. M., Definitive Molecular Level Characterization of
Defects in UiO-66 Crystals. Angew. Chem. Int. Ed. 2015, 54 (38), 11162-11167.

20. Tsuruoka, T.; Furukawa, S.; Takashima, Y.; Yoshida, K.; Isoda, S.; Kitagawa, S., Nanoporous nanorods fabricated by coordination modulation and oriented attachment growth. Angew. Chem. Int. Ed. 2009, 48 (26), 4739-43.

21. Wißmann, G.; Schaate, A.; Lilienthal, S.; Bremer, I.; Schneider, A. M.; Behrens, P., Modulated synthesis of Zr-fumarate MOF. Microporous and Mesoporous Materials 2012, 152, 64-70.

22. Schaate, A.; Roy, P.; Godt, A.; Lippke, J.; Waltz, F.; Wiebcke, M.; Behrens, P., Modulated synthesis of Zr-based metalorganic frameworks: from nano to single crystals. Chem.-Eur. J. 2011, 17 (24), 6643-51.

23. Zahn, G.; Zerner, P.; Lippke, J.; Kempf, F. L.; Lilienthal, S.; Schröder, C. A.; Schneider, A. M.; Behrens, P., Insight into the mechanism of modulated syntheses: in situ synchrotron diffraction studies on the formation of Zr-fumarate MOF. CrystEngComm 2014 16 (39), 9198-9207.

24. Firth, F. C. N.; Cliffe, M. J.; Vulpe, D.; Aragones-Anglada, M.; Moghadam, P. Z.; Fairen-Jimenez, D.; Slater, B.; Grey, C. P., Engineering new defective phases of $\mathrm{UiO}$ family metal-organic frameworks with water. Journal of Materials Chemistry A 2019.

25. Coelho, A. A.; Evans, J.; Evans, I.; Kern, A.; Parsons, S., The TOPAS symbolic computation system. Powder Diffr. 2011, 26 (S1), S22-S25.

26. Gómez-Gualdrón, D. A.; Moghadam, P. Z.; Hupp, J. T.; Farha, O. K.; Snurr, R. Q., Application of Consistency Criteria To Calculate BET Areas of Micro- And Mesoporous Metal-Organic Frameworks. J. Am. Chem. Soc. 2016, 138 (1), 215-224

Insert Table of Contents artwork here

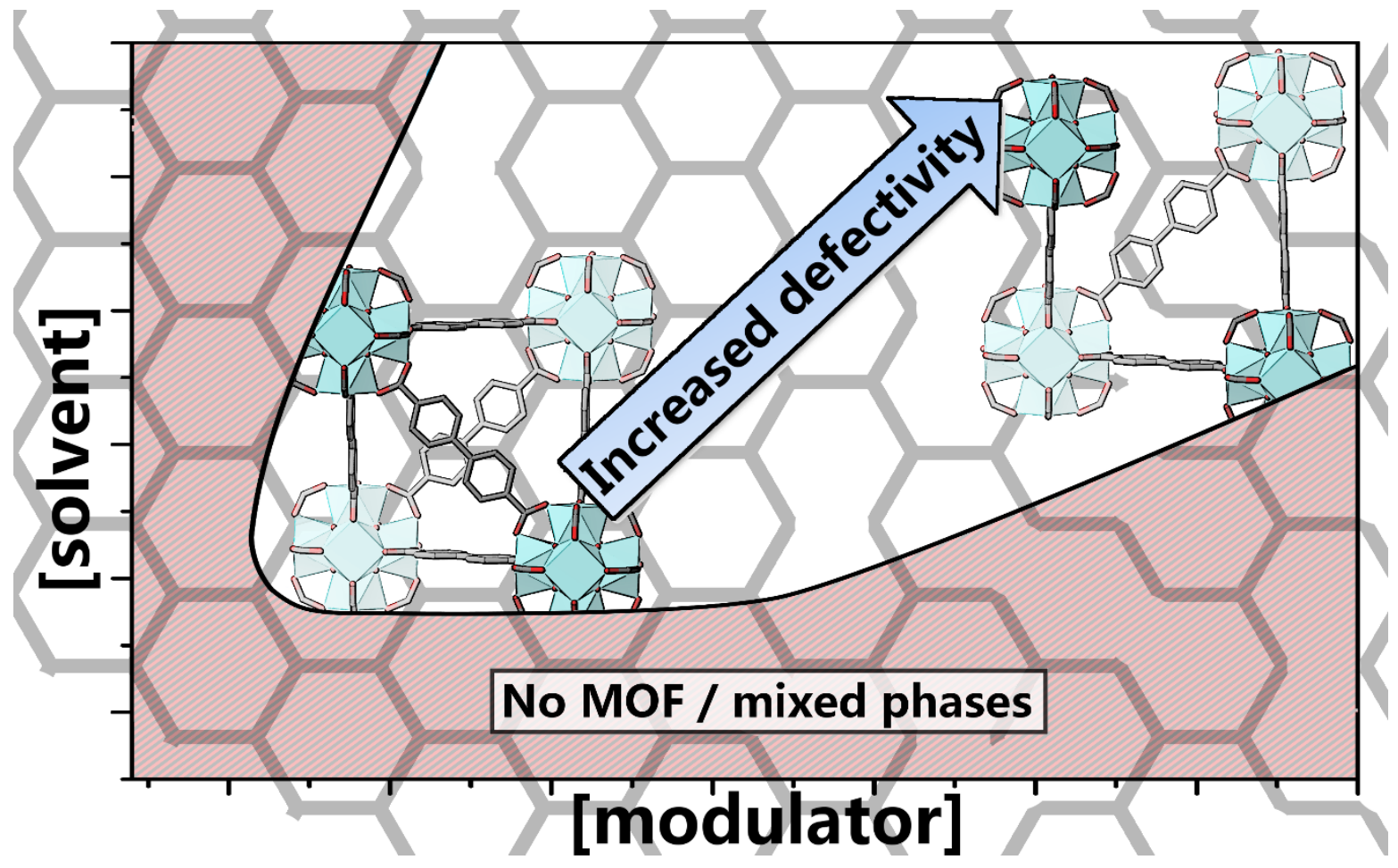

Conventional synthesis methods of Zr-MOFs often result in MOFs with high concentrations of linker vacancy defects. In UiO-67, the degree of defectivity can be controlled by adjusting the ratios of reagents, modulator and solvent in the synthesis. 